\title{
Integrative Oncology: A Paradigm Shift in Cancer Care
}

\author{
Richard T. Lee, MD
}

Extensively trained in the fields of integrative medicine and oncology, Richard T. Lee, MD, is one of the first physicians in the United States to hold a unique position as a professor in integrative oncology. Committed to his calling, Dr. Lee's research and clinical and educational endeavors are not only advancing the field of oncology, but also shifting our view of cancer care and the essential need for an integrative approach.

Q: How important is an integrative approach in the field of oncology today? Is there a need for a paradigm shift in the way that we, as clinicians, view people with cancer?

Richard T. Lee, MD: Integrative oncology adds significant value to the field of cancer medicine, especially in the areas of survivorship and palliative care, and the field is growing in importance. Cancer centers throughout the world are recognizing this, and most major cancer centers in the United States now have integrative services for patients. The recent opioid epidemic - especially here in Ohio where we have some of the highest rates of overdoses - is forcing the field of medicine and cancer care specifically to understand that medications are not always the answer. There are nonmedication approaches to managing pain, anxiety, and stress, and this is a great entrée for integrative oncology to help address these conditions and to look at integrative approaches for symptom management. The opioid epidemic creates urgency for us to open our eyes to the use and availability of other effective therapies.

There has also been increasing recognition among oncologists that, when seeing patients, we need to do a better job of addressing the whole person. The Institute of Medicine released a publication on cancer care for the whole person and emphasized the importance of addressing psychosocial needs. ${ }^{1}$ Within the field, there is now greater acknowledgment that we need to have a more integrative approach, and this is where the field of integrative oncology can significantly help people with cancer.

People in the field of integrative oncology can make a difference in the lives of patients in both inpatient and outpatient settings. Here at University Hospital's Seidman Cancer Center, Cleveland, Ohio, for example, we have begun providing acupuncture in the chemotherapy infusion suites to help address stress, anxiety, and nausea and vomiting. In addition, in my integrative oncology consultation service at the cancer center, I receive many referrals of patients who have chronic pain or chronic neuropathy and are interested in a nonmedication approach. Often these people are already taking too many medications or they have tried all of the standard treatments and have not experienced significant symptom reduction. They are relieved to learn about additional therapies such as acupuncture, which can help ease their symptoms. Seeing these patients experience benefits - sometimes within just a few weeks - is very satisfying for both the patient and the clinician. In my clinical experience, large percentages of patients are interested in and would benefit from an integrative approach.

I recently saw a young patient with metastatic colon cancer who started incorporating yoga and meditation into everyday life. This person had a high-stress job and a lot of stress in general, but instead of trying to treat this problem with an antianxiety medication, we explored the value of practicing mind-body therapies such as yoga and meditation.

There are so many integrative therapies that clinicians can offer patients, which have very few side effects, and one does not have to worry about addiction or overdose. In our oncology center, we turn to mind-body therapies, acupuncture and have well-established art and music therapy programs through Connor Integrative Health Network at University Hospitals, Cleveland, Ohio. We also refer many patients to The Gathering Place, Beachwood, Ohio, which is an important community resource that provides free complementary therapies and programming for patients and families affected by cancer.

Q: What are some cutting-edge ways in which the field of integrative oncology has especially evolved during the past decade?

Dr. Lee: Many exciting things are happening to strengthen and advance the field of integrative oncology. For example, it is great to see practice guidelines emerge regarding the use of complementary therapies for people with cancer. This recent effort has mainly been driven by the Society for Integrative Oncology (SIO), which received grants from the Samueli Foundation, to continue to work on additional practice guidelines and has been spearheaded by Heather Greenlee, ND, PhD, MPH, Suzie Zick, ND, MPH, and Debu Tripathy, MD. 
The development of practice guidelines has been a huge step forward for the field. Last year, the guidelines were coapproved by the American Society for Clinical Oncology (ASCO), which is the world's major oncology organization. To receive that level of collaboration on practice guidelines from ASCO is a huge accomplishment for the field. The National Comprehensive Cancer Network guidelines are often considered the standard of care guidelines for cancer treatment in general. If you look at the symptom management guidelines, they are now incorporating therapies such as acupuncture, mindfulnessbased stress reduction, and yoga. The American College of Physicians' guidelines for chronic low-back pain have started to include acupuncture and mind-body techniques, as well, so we are seeing incorporation of these types of therapies in wellrespected national standard of care guidelines that already exist. Complementary therapies are truly being integrated into a patient's care plan, so that is wonderful.

I also believe that the research in integrative oncology is improving. We are seeing increasing randomized controlled trials including treatments such as acupuncture, meditation, yoga, and music and art therapy. To see researchers conducting these higher quality clinical trials is essential to advancing the field.

Another interesting thing that is happening in the field is that researchers are increasingly studying the mechanisms of how complementary therapies actually help patients. For example, with acupuncture, researchers are looking at issues such as pain signaling and natural endorphin release. There are fascinating imaging studies using functional MRI, trying to localize activation within the brain of certain acupuncture points. These types of scientific endeavors help the field move forward as we begin to understand these therapies better so that we can apply them to the right patients and even make these therapies more effective.

Q: How has your interest and career in integrative oncology evolved? When did you first become interested in this area of medicine?

Dr. Lee: The seeds of interest were planted during my childhood. My parents are both from Taiwan, and I remember my father having an acupuncture doll and needles in his medical clinic. I have always been fascinated by the idea of acupuncture as a tool for healing. Also, my grandmother lived with us, and growing up I remember her going to the Chinese medicine herbalist to buy herbs and then she would stew them. There was a unique odor throughout our home while she was boiling these Chinese herbs, which she was making to improve her health. So these were some of the many small exposures to Oriental medicine during my youth.

My real entrée into the field, however, started during an open year between residency and fellowship. I pondered what to do with that year and decided I would go back to Asia to learn more about Traditional Chinese Medicine (TCM). After completing my residency at Stanford School of Medicine, Palo Alto, California, I was very fortunate to receive a Fulbright Scholarship to travel to Taiwan and learn about TCM with a focus on acupuncture. I dove right into the experience and spent the first couple of months learning Chinese so that I could improve my language skills before starting classes. I then attended China Medical University in Taichung, Taiwan, which is very unique in that when students attend for a medical degree, they receive both a degree in Western medicine and in Chinese medicine. This experience opened my eyes to the power of modalities such as acupuncture to help patients, and I liked the fact that the University embraced both Western and Eastern medical systems. I observed that if my professors felt something was very serious and that Chinese medicine was not the right approach, they would say, "You need to see a Western doctor because this seems like a serious problem." They were able to see the value of when their approach was appropriate or not. This dual approach was one of the major takeaway points from my experience.

I also spent time at an international acupuncture-training center in Beijing, China, and received additional exposure to learning about TCM theory and diagnosis. Ultimately, I learned that there is a lot more commonality than people realize between Western and Eastern systems of health care. People often view these as two very different systems; however, I think they have many of the same concepts. In both systems, as clinicians we talk with the patient, ask questions, and begin to determine what is happening. Then we seek to discover the underlying causes and reach a diagnosis. Based on that diagnosis, we treat the patient. The fundamentals are the same. Of course, each approaches the problem with different types of treatments, but ultimately it is the same concept of wanting to understand the diagnosis and underlying cause.

After this year of exploration and learning, I returned from that experience to begin my hematology/oncology fellowship and realized that I wanted to bring the things that I had learned into cancer care. This was when I actually started to practice integrative oncology. During this time, I attended SIO meetings, which was a great experience and provided a deeper realization that integrative oncology was a developing field and that I could contribute to this area. Such conferences can be very helpful for guiding clinicians in their work. I am the past treasurer for SIO and have cochaired two SIO conferences.

Much of what integrative oncology has to offer people with cancer focuses on symptom management and quality of life, and I felt I needed to understand this better. Therefore, I also completed a Palliative Medicine Fellowship at Northwestern University, Chicago, Illinois. This training was very helpful with regard to understanding more about when complementary therapies are most appropriate in cancer symptom management.

When I completed my training, I became the director for the Integrative Medicine Center at MD Anderson Cancer Center in Houston, Texas. It was a great opportunity and like the Fulbright scholarship, it happened at the right time. Then in 2016, I was recruited to be considered for the Helen Moss FoundationSchoff Family Professor of Integrative Oncology at Case Western Reserve University School of Medicine, Cleveland, Ohio. I thought it was a great match and, of course, the rest is history. 
Q: Tell us about your unique position as the Helen Moss Foundation-Schoff Family Professor of Integrative Oncology.

Dr. Lee: It is a unique position in that I believe it is currently the only physician professorship that is specific to integrative oncology. Helen Moss, the primary donor and visionary for the position, wanted an oncologist to fill this post. In that sense, it was forward thinking and unique to create this professorship within a top 25 medical school such as Case Western Reserve University School of Medicine, Cleveland, Ohio. Very few such positions exist right now, and I believe this is the only one that is designated to be filled by a physician oncologist. The professorship supports my ability to pursue academic endeavors around research and education. I conduct research, and I teach the medical students and generally expand the visibility of the importance of integrative oncology in the field of medicine.

I am also the director for the Supportive and Integrative Oncology Program at University Hospital's Seidman Cancer Center, and this position is focused more broadly on building and coordinating supportive oncology services. In addition, I see patients as a medical oncologist, and my clinical focus is on gastrointestinal cancers, and particularly liver cancer.

Q: Please share with us some current and interesting research endeavors and findings in the field of integrative oncology.

Dr. Lee: In our cancer center, we are just opening up a clinical trial looking at acupuncture for chemotherapy-induced peripheral neuropathy, and we have plans to look at the benefits for people who have postoperative ileus as well. We also have another protocol we are working on, potentially using acupuncture to ease symptoms of pain and anxiety in patients undergoing bone marrow biopsy. In addition, we are conducting a meditation study, looking at how we can optimize meditation techniques in people with cancer. As one can see, we are involved in exciting and promising research endeavors.

We have also been doing survey work looking at, for example, patients' levels of interest in different types of integrative therapies such as acupuncture, massage, and music therapy. One of the things we have learned so far is that, in general, patients seem to be most familiar with massage therapy and are least familiar with therapies such as acupuncture. A lack of education was found to be a barrier to knowing about such treatments, so much of our effort is centered on educating both patients and clinicians about these services and the potential benefits. Another barrier we identified is lack of access to integrative therapies and that the costs of these services can be prohibitive. To address access issues, within our cancer center and together with Connor Integrative Health Network, we are subsidizing the costs of some of these therapies. For example, we have implemented a sliding scale for acupuncture, and patients who qualify can actually receive free acupuncture. We want to be able to make sure that patients who can benefit from these therapies have access. Access is a major issue that we must address in the field of integrative medicine.
We also have interesting data looking at meditation. My colleagues and I conducted a pilot study wherein we collected data from expert meditators and nonmeditator volunteers. We collected a lot of data, including electroencephalography data, and we noticed that before a stressor was introduced, both the expert meditators and nonmeditators could achieve a relaxed state. However, after the stressor, we saw a significant difference. Compared with the nonmeditators, the expert meditators seemed to be able to return to a relaxed state sooner. In this study, we are demonstrating objectively that by practicing mind-body techniques, people may recover more quickly from a stressful event. This is obviously very pertinent for people dealing with cancer who often experience lots of stress, and these practices can help build resiliency.

My other main area of research is investigating natural plant products in the laboratory. Currently, my colleagues and I have been studying effects of mistletoe and mushroom extracts. In the laboratory, we have found that these extracts appear to have anticancer effects, and we are trying to determine the active compounds and the active mechanisms of action. We just published our first article in Scientific Reports, demonstrating mistletoe extracts' potential anticancer activity, both in vitro and in vivo mouse models. ${ }^{2}$ We may have identified the active compound of mistletoe, which is lectin, and we are further studying how it works, hopefully, with the goal of moving forward with new treatment options for people with cancer.

Q: In terms of the future, what do you hope to accomplish in your unique position as a professor of integrative oncology?

Dr. Lee: My integrative oncology colleagues and I here at the cancer center are focused on a deconstructionist optimization approach. In other words, we are very interested in breaking down integrative therapies and understanding the various components. For example, we want to better understand meditation's different components. There is a deep breathing component, a mindfulness component, and a focused attention component, and we want to look at how each of those components helps contribute to the benefits of utilizing such a therapy. Then, as we deconstruct it and understand it better, perhaps we can reconstruct meditation in the most optimal and effective way for our patients. Essentially we are asking what makes a particular therapy work, and how do we make it better and more optimal and effective? This is important work now and for the future.

We take a similar approach in our study of natural plant products - we want to break the components down and understand what works. Is it a single compound that is exerting its effects or is it more than one compound? How do we make a natural plant extract even more effective for patients? For the long term, we are looking at integrative therapies and what kind of impact we hope to have on the field.

Ultimately, I believe we can create better access to integrative medicine therapies for the people who need them the most. This will require insurance companies to view reimbursement 


\section{To Contact Dr. Richard T. Lee}

Richard T. Lee, MD

Director of Supportive and Integrative Oncology University Hospital's Seidman Cancer Center

Cleveland, Ohio

Email: Richard.T.Lee@case.edu

Website: https://case.edu/cancer/research/case-center-

integrative-oncology

for such therapies for pain the same way that they view the benefits of a medication for high blood pressure, which is covered. If we have enough high-quality research and a solid body of evidence, then insurance companies will realize that not only are these effective therapies, but also it becomes cost-effective to treat patients' pain with yoga or meditation or acupuncture. Then patients will not have to worry about the barriers they deal with now to try to receive integrative therapies.

The number of symptoms that can be addressed by integrative therapies is growing. Moving forward, it is important that we embrace this knowledge, understand our patients' perspectives, and then continually ask how we can best meet a person's diverse needs as they go through cancer treatment.

\section{References}

1. Adler NE, Page AE, and Institute of Medicine (US) Committee on Psychosocial Services to Cancer Patients/Families in a Community Setting. Cancer Care for the Whole Patient Meeting Psychosocial Health Needs. Washington, DC: National Academies Press, 2008.

2. Yang P, Jiang Y, Pan Y, et al. Mistletoe extract Fraxini inhibits the proliferation of liver cancer by down-regulating c-Myc expression. Sci Rep 2019;9:6428.

Richard T. Lee, MD, is director of supportive and integrative oncology at University Hospital's Seidman Cancer Center, Cleveland, Ohio and the Helen Moss Foundation-Schoff Family Professor of Integrative Oncology, Case Western Reserve University School of Medicine, Cleveland, Ohio. He is also an associate professor in the Division of Hematology and Oncology, Case Western Reserve University School of Medicine, Cleveland, Ohio.

To order reprints of this article, contact the publisher at (914) 740-2100. 